\title{
Unique lamina propria stromal cells imprint the functional phenotype of mucosal dendritic cells
}

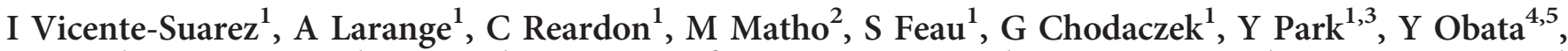 \\ R Gold $^{1}$, Y Wang-Zhu ${ }^{1}$, C Lena ${ }^{1}$, DM Zajonc ${ }^{2}$, SP Schoenberger ${ }^{1}$, M Kronenberg $^{1}$ and H Cheroutre ${ }^{1}$
}

Mucosal dendritic cells (DCs) in the intestine acquire the unique capacity to produce retinoic acid (RA), a vitamin A metabolite that induces gut tropism and regulates the functional differentiation of the T cells they prime. Here, we identified a stromal cell (SC) population in the intestinal lamina propria (LP), which is capable of inducing RA production in DCs in a RA- and granulocyte-macrophage colony-stimulating factor (GM-CSF)-dependent fashion. Unlike DCs, LP SCs constitutively expressed the enzymatic machinery to produce RA even in the absence of dietary vitamin $A$, but were not able to do so in germ-free mice implying regulation by microbiota. Interestingly, DCs promoted GM-CSF production by the SCs indicating a two-way cross-talk between both cell types. Furthermore, RA-producing LP SCs and intestinal DCs localized closely in vivo suggesting that the interactions between both cell types might have an important role in the functional education of migratory DCs and therefore in the regulation of immune responses toward oral and commensal antigens.

\section{INTRODUCTION}

Genetic regulation by vitamin A is involved in multiple biological processes such as embryonic development, vision, and immunity. ${ }^{1}$ To exert this regulatory role, vitamin $\mathrm{A}$ is oxidized into its active form by retinol dehydrogenases followed by retinal dehydrogenases (RALDH). ${ }^{2}$ The product of this metabolism, retinoic acid (RA), binds to nuclear RA receptors (RARs), and together drive the transcription of target genes that contain RA responsive elements (RAREs) within their promoters. $^{3}$

RA is especially critical for the regulation of immune responses within the digestive tract, thereby controlling functional T-cell differentiation and directing lymphocyte migration toward the intestine. ${ }^{4,5}$ Accordingly, dendritic cells (DCs) in the lamina propria (LP), Peyer's patches, and mesenteric lymph nodes (MLNs), but not spleen- or peripheral lymph nodes, express RALDH and produce RA. ${ }^{4}$ Under steady-state conditions RA contributes to dampening effector responses by blocking Th1 and Th17 differentiation and enhancing immunoglobulin A production as well as transforming growth factor $\beta$ (TGF $\beta$ )-driven-Treg differentiation. ${ }^{5-8}$ In contrast, under inflammatory conditions, in the presence of interleukin (IL)-15, RA exacerbates immune pathology by driving IL-12 production. ${ }^{9}$ These observations place RA as a master regulator of tolerogenic as well as inflammatory immune responses especially in the digestive tract.

CD103-expressing migratory DCs that transport and present gut-derived antigens to naïve T cells in the MLNs typically produce RA, ${ }^{10,11}$ suggesting that they gain this hallmark feature during their education and maturation in the intestinal compartment. In agreement with this notion, MLNs of mice deficient for the chemokine receptor 7 (CCR7), which is required for efficient lymph node homing, are lacking mucosal RA-producing DCs. ${ }^{11}$

Despite this, it is not known how migratory DCs acquire RALDH activity, and the relevance of different factors such as cytokines or the presence of commensals has been alternatively supported or rejected by different studies. ${ }^{12,13}$ Nonetheless, there is substantial evidence demonstrating that RA signaling is required and might be sufficient for the initial induction of

\footnotetext{
${ }^{1}$ Division of Developmental Immunology, La Jolla Institute for Allergy and Immunology, La Jolla, California, USA. ²Division of Cell Biology, La Jolla Institute for Allergy and Immunology, La Jolla, California, USA. ${ }^{3}$ Division of Integrative Biosciences and Biotechnology, Pohang University of Science and Technology, Pohang, Korea. ${ }^{4}$ Division of Mucosal Barriology, International Research and Development Center for Mucosal Vaccines, The Institute of Medical Science, The University of Tokyo, Tokyo, Japan and ${ }^{5}$ Laboratory for immune regulation, Graduate School of Medicine, Chiba University, Chiba, Japan. Correspondence: H Cheroutre (hilde@liai.org) 
RALDH in DCs, not in the least the observation that vitamin A-deficient (VAD) mice lack RALDH ${ }^{+}$DCs in the LP and $\mathrm{MLNs}^{14,15}$ and that exposure to exogenous RA was sufficient to induce RALDH activity in DCs as shown in some reports. ${ }^{16,17}$

To identify in vivo the intestinal cell type that has the capacity to educate migratory DCs and to elucidate the mechanisms that lead to RA production by $\mathrm{CD} 103^{+}$DCs, we dissected and characterized various cell subsets from the intestinal LP and identified a stromal cell (SC) population capable of imprinting DCs with RALDH activity. These SCs are an abundant component of the intestinal LP and might represent a direct source of RA. Importantly, we show that these SCs are in close contact with CD103-expressing DCs and that this interaction conversely promotes granulocyte-macrophage colony-stimulating factor (GM-CSF) secretion by the SCs, which in addition to RA, is absolutely required for effective RALDH induction in the DC compartment. Finally, we also found that, unlike DCs, the constitutive RALDH expression by LP SCs is independent of RA, whereas it did require the presence of the microbiota. Our findings therefore identified an RA-producing LP SC as a direct sensor of the gut environment and an important regulator of the functional maturation of mucosal DCs. The results also demonstrate an unexpected two-way cross-talk between these SCs and the DCs that might have an important role in controlling the tolerogenic or inflammatory nature of the mucosal immune response.

\section{RESULTS}

\section{A subset of intestinal LP SCs produce RA}

Mucosal migratory DCs are marked by the expression of RALDH and the capacity to produce RA, which modulates the immune response. ${ }^{4-6} \mathrm{RA}$ is required to induce RALDH expression in mucosal DCs suggesting that they might be initially exposed to RA during their development in the LP. ${ }^{14}$ Nevertheless, the source of RA as well as the education process that grants mucosal DCs their unique immune regulatory capacity are still poorly understood. To identify potential local sources of RA, we analyzed LP cells using aldefluor, a fluorescent RALDH substrate that marks those cells which have capacity to produce RA. Interestingly, a significant population of CD45-negative cells displayed high RALDH activity (Figure 1a upper left panel). The majority of these RALDHpositive cells were negative for the epithelial cell marker Epcam (CD326) (Figure 1a upper right panel), suggesting that in addition to intestinal epithelial cells, which are known to constitutively express RALDH ${ }^{4,18,19}$, there is a subset of cells in the LP that also produces RA. RALDH activity among the SCs was confined to a specific population that shows remarkable similarities with the well-characterized fibroblastic reticular cells. These similarities included the expression of podoplanin (Pdpn) in the absence of CD31 (Figure 1a lower left panel), and they expressed ICAM-1 alone or in combination with VCAM-1 (Figure 1a lower right panel). RALDH expression in sorted CD31 ${ }^{-} \mathrm{CD}^{-} 5^{-}$Epcam $^{-}$Pdpn $^{\text {hi }}$ small intestinal (SI) LP SCs was confirmed by quantitative PCR. Unlike intestinal epithelial cells, which mainly expressed aldh1a1, $\mathrm{Pdpn}{ }^{+} \mathrm{CD} 31^{-}$SCs expressed high levels of all three RALDH encoding genes, aldh1a1, -2 , and -3 (Figure $\mathbf{1 b}$ ). To assess whether aldefluor-positive SCs also produced RA, supernatants from overnight cultures of these cells were used to stimulate a reporter cell line that produces $\beta$-galactosidase under the control of a RARE. ${ }^{20}$ In agreement with active secretion of RA, supernatants increased $\beta$-galactosidase production relative to media as measured through a colorimetric reaction (Figure 1c left panel), and the absorbance values obtained were similar to those of media supplemented with $4 \mathrm{~nm}$ RA (Figure 1c right panel).

To test whether ALDH expression by $\mathrm{Pdpn}^{+} \mathrm{CD} 31^{-}$SCs was RA dependent, we compared aldefluor staining in LP cells isolated from VAD and vitamin A-sufficient (VAS) mice (Figure 1d). We did not find a significant difference between both conditions suggesting that, in contrast to migratory DCs $^{14,15}$ (Supplementary Figure S1 online), RA was not required for the induction or maintenance of the RA metabolism machinery in LP SCs. Interestingly, RALDH expression in the LP SCs, but not in the DCs, did depend on the presence of the intestinal microbiota as aldefluor staining was practically undetectable in SCs from germ-free mice (Figure 1e; Supplementary Figure S1).

RALDH $^{+}$LP SCs directly interact with $\mathrm{CD} 103^{+} \mathrm{DCs}$ in vivo To investigate the location of RALDH-positive SCs within the LP compartment, aldefluor staining was directly performed in live SI explants in which the epithelial layer was removed to allow penetration of the reagent into the intestinal mucosa. Aldefluor bright cells were numerous and distinct from the $\mathrm{CD} 31^{+}$endothelial cells and platelets, which marked the blood vessels in the villi (Figure 2a). In order to examine the proximity of these RA-producing SCs to DCs in the LP, we co-stained tissue explants with aldefluor and the DC marker, CD11c. Aldefluor bright SCs were clearly noticeable in the vicinity of the $\mathrm{CD} 11 \mathrm{c}^{+}$cells but not in the presence of the RALDH inhibitor DEAB, indicating that the fluorescence observed correlated with RALDH activity (Figure 2b). More importantly, merged staining of CD11c and aldefluor at the interface between RA-producing $\mathrm{SCs}$ and $\mathrm{CD}_{11 \mathrm{c}^{+}}$cells indicated that there was direct contact between the two cell types (Figure 2c). Remarkably, many of the $\mathrm{CD} 11 \mathrm{c}^{+}$cells that directly contacted the aldefluor bright SCs were also dim for aldefluor staining (Figure 2c). CD11c expression is not exclusive for DCs but co-staining of CD11c together with CD103 faithfully identifies migratory DCs. ${ }^{10,11}$ Contact between aldefluor bright SCs and $\mathrm{CD} 11 \mathrm{c}^{+}$cells that co-stained for CD103 was particularly striking, suggesting that in vivo there is a direct cross-talk between RA-producing LP SCs and migratory DCs that acquire the regulatory capacity to produce RA (Figure 2c).

\section{RALDH $^{+}$LP SCs induce RALDH activity in splenic DCs}

To test whether aldefluor ${ }^{+}$LP SCs have the capacity to induce RA production in DCs, we sorted SI Pdpn ${ }^{+}$CD31 ${ }^{-}$SCs and cocultured them for $24 \mathrm{~h}$ with splenic DCs. After conditioning 


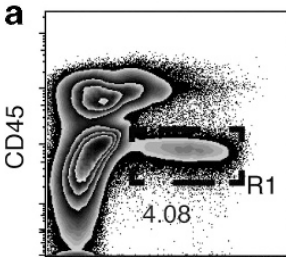

Aldefluor

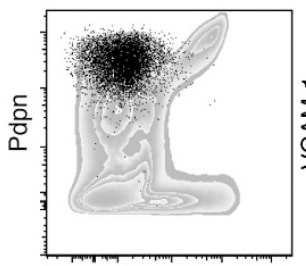

CD31

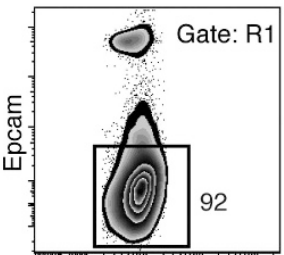

CD45

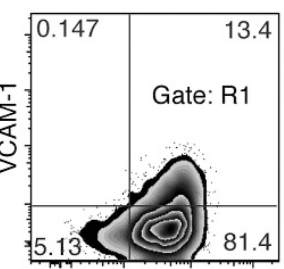

ICAM-1
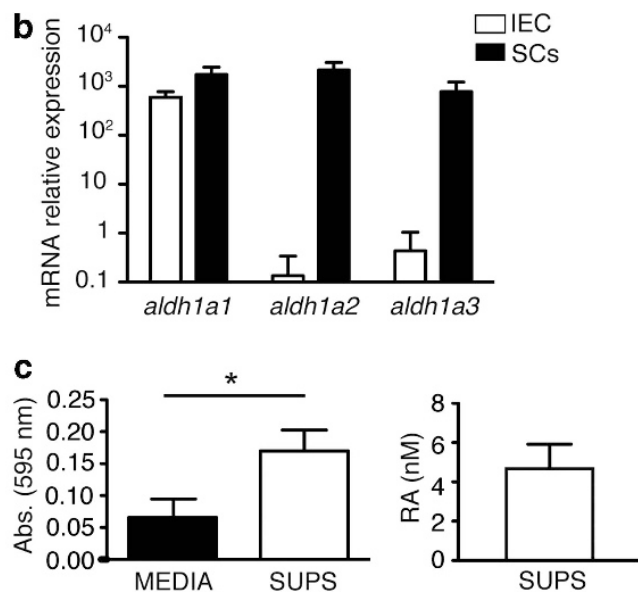

d

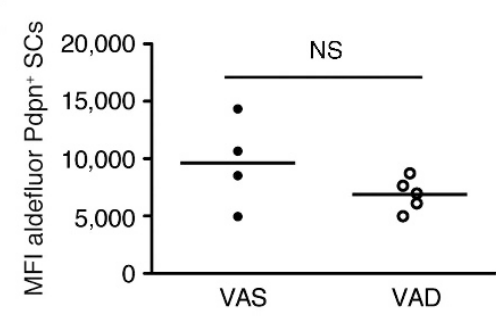

e

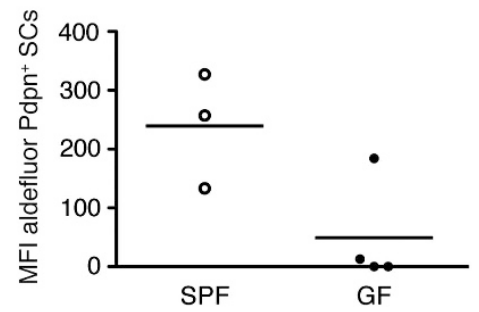

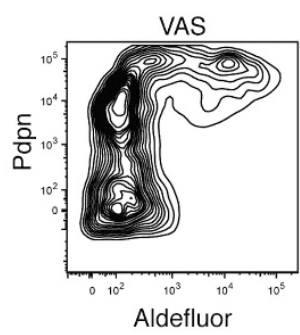
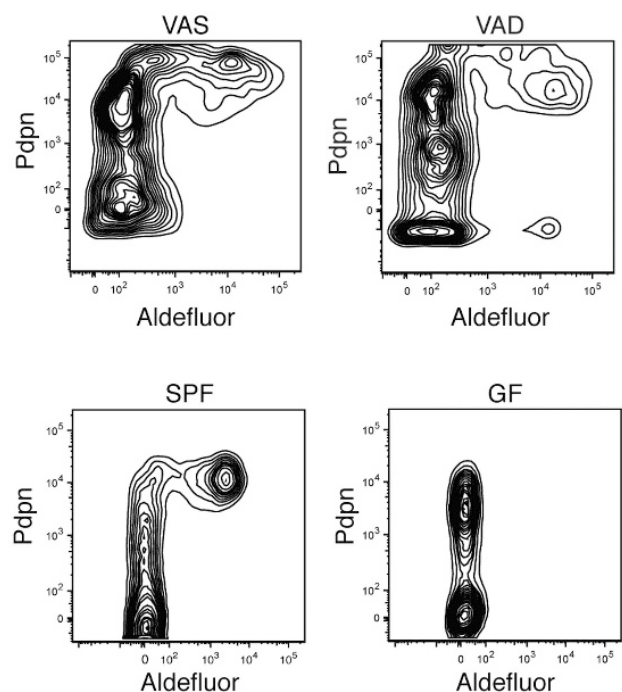

Figure 1 A subset of lamina propria (LP) stromal cells (SCs) have the capacity to produce retinoic acid (RA). (a) Analysis of RALDH activity using aldefluor in single-cell suspensions prepared from small intestinal (SI) LP and assessed by flow cytommetry for the indicated markers. Left lower panel displays CD $45^{-}$aldefluor ${ }^{+}$cells (gate R1, black color) within the whole SI LP SC population (gray color). Data show a representative experiment of $>5$ independent experiments. (b) messenger RNA (mRNA) levels of the RA-producing enzymes RALDH1 (aldh1a1) RALDH2 (aldh1a2), and RALDH3 (aldh1a3) were measured by quantitative real-time PCR in sorted CD45 ${ }^{-}$Epcam ${ }^{-}$CD31- ${ }^{-}$dpn $^{+}$cells (SCs, black bars) and CD45 ${ }^{-}$Epcam ${ }^{\text {hi }}$ cells (intestinal epithelial cell, white bars). Data shown correspond with RNA from three independent experiments that were quantified at the same time. (c) Supernatants from sorted SCs were used to stimulate F9-RARE-lacZ cells and $\beta$ galactosidase production by these cells was measured using a colorimetric reaction. Left panel compares the absorbances obtained when F9-RARE-lacZ cells were left untreated (black bar) or supernatants were added to the culture (white bar); right panel shows an estimation of the RA cc present in the supernatants. Represented is the average \pm s.d. from three independent experiments pooled together ( ${ }^{\star} P<0.05 t$-test non paired). (d) Aldefluor staining of $C D 45^{-} P d p n{ }^{+} S C s$ was compared between specific pathogen-free (SPF) and germ-free mice (GF) or (e) between vitamin A-sufficient (VAS) and vitamin A-deficient (VAD) mice. Shown is a representative experiment out of three independent experiments $(P=0.18$, NS, not significant $t$-test non paired). Sups, suspension.

with Pdpn ${ }^{+} \mathrm{CD} 1^{-}$SCs, splenic DCs became aldefluor high (Figure 3a). Furthermore, compared with unconditioned DCs, SCs-cocultured DCs suppressed Th17 differentiation and induced the gut homing receptors $\alpha 4 \beta 7$ and CCR9 (Figure 3b; Supplementary Figure S2a), both typical effects of RA.,

The effects were blocked when the RALDH inhibitor DEAB was added, ruling out that they were caused by a carryover of RA from the SCs-coculture, as active production of the metabolite by RALDH in the DCs was required (Figure 3b). Surprisingly, SCs-cocultured DCs did not significantly increase TGF- $\beta$-mediated Foxp $3^{+}$Treg differentiation ${ }^{6}$ in vitro (Supplementary Figure S2b, upper panels).
However, because SCs-conditioned DCs showed signs of activation as indicated by the increased expression of activation markers including, CD86 (Supplementary Figure S2c), it is likely that Foxp $3^{+}$Treg differentiation is suppressed under these coculture conditions. ${ }^{8}$ In support of this, when RA production by the DCs was blocked with DEAB, SCs-conditioned DCs induced much less Foxp $3^{+}$Treg differentiation compared with untreated DCs (Supplementary Figure S2b, lower panels), indicating that RA produced by the activated SCs-conditioned DCs did enhance the Foxp $3^{+}$Treg differentiation. Interestingly, when RA production was blocked, SCsconditioned DCs induced more Th17 differentiation than 

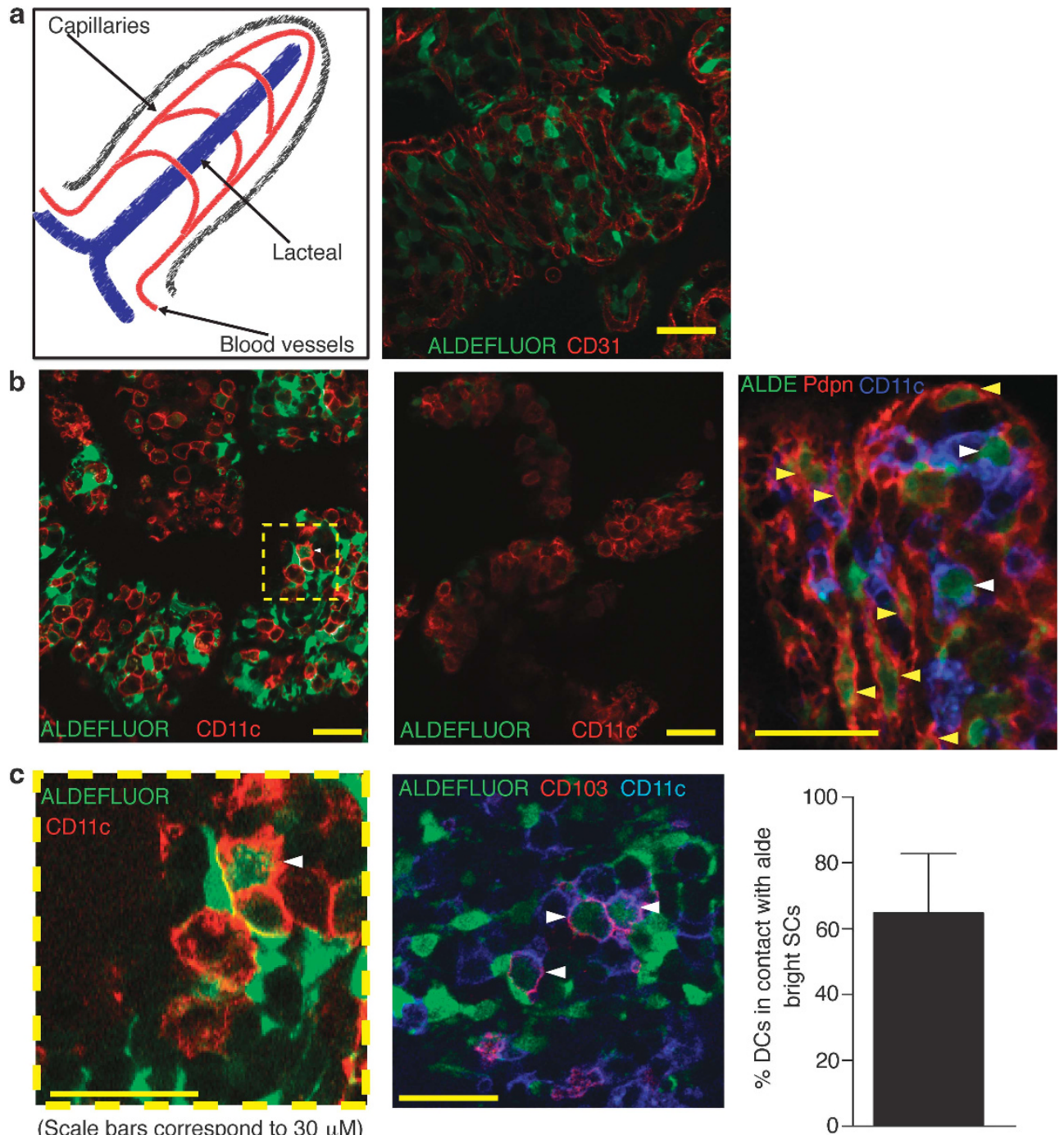

(Scale bars correspond to $30 \mu \mathrm{M}$ )

Figure 2 Aldefluor-positive stromal cells (SCs) are abundant within the intestinal lamina propria and locate closely to intestinal dendritic cells (DCs). (a-c) Confocal imaging of aldefluor-stained live small intestinal explants after intestinal epithelial cell removal. Aldefluor staining was followed by immunofluorescence with the indicated antibodies, anti-CD31 (a), anti-CD11c (b), or anti-CD11c plus anti-podoplanin (b, right panel) or plus anti-CD103 (c, right panel). In the indicated images (b, central panel) explants were stained with aldefluor in the presence of the RALDH inhibitor DEAB. White arrowheads point out aldefluor ${ }^{+}$DCs in close contact with aldefluor ${ }^{+}$SCs, yellow arrowheads indicate podoplanin ${ }^{+}$aldefluor ${ }^{+}$cells. Quantification shows the percentage of $\mathrm{CD} 11 \mathrm{c}^{+} \mathrm{CD}_{103^{+}}$cells in direct contact with aldefluor bright lamina propria cells from $>10$ different images. Scale bar $=30 \mu \mathrm{M}$.

untreated DCs (Figure 3b SCs cond. + DEAB), suggesting that the LP SCs also enhanced the functional priming capacity of the DCs they interacted with.

To investigate whether soluble factors released by $\mathrm{Pdpn}^{+}$ $\mathrm{CD} 31^{-}$SCs were able to educate DCs, we conditioned splenic DCs for $24 \mathrm{~h}$ with media harvested from Pdpn ${ }^{+}$CD $31^{-}$SCs overnight cultures. Splenic DCs stimulated with these supernatants specifically upregulated aldh1a2 expression (Figure 3c), which is the RALDH isoform typically expressed by the mucosal migratory DCs.

Furthermore, a global RA receptor antagonist (BMS 204493) blocked RALDH expression in DCs induced either by direct contact with Pdpn ${ }^{+}$CD31 ${ }^{-}$SCs (Figure 3d, left panel) or by SC-derived supernatants (Figure 3d, right panel), indicating that RALDH induction in DCs depended on RA signaling. Overall, these data demonstrate that $\mathrm{LP} \mathrm{Pdpn}{ }^{+} \mathrm{CD} 31^{-}$SCs can induce RA-dependent transcriptional activation of aldh1a2 in DCs, which leads to the expression of RALDH2 and the production of RA, a hallmark feature of the migratory mucosal $\mathrm{CD}_{103}{ }^{+} \mathrm{DC}$.

\section{GM-CSF produced by the LP SCs is required for the RA-induced RALDH expression in DCs}

To investigate whether RA secreted by the SCs is sufficient to induce RALDH expression in spleen DCs, various concentrations of RA were added to overnight cultures of spleen DCs. Unlike supernatants from the RALDH ${ }^{+}$LP SCs, RA by itself was inefficient to induce RALDH expression in the overnight 
a

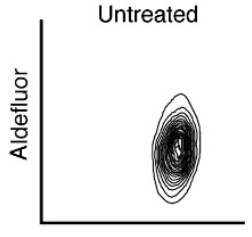

b
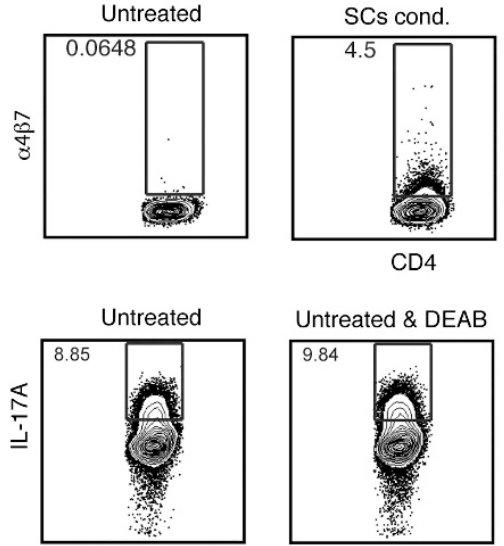

CD4

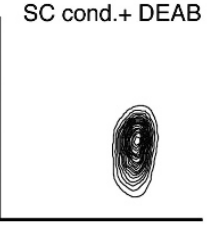

SCs cond \& DEAB.
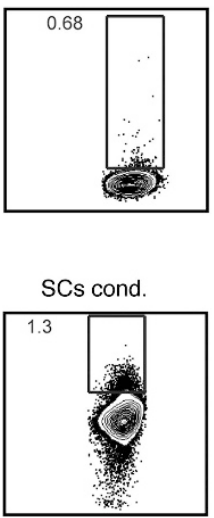

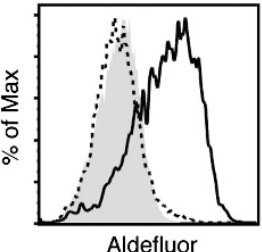

Aldefluor $\square$ Untreated

- SCs cond.

-.. SCs cond.

+ DEAB c

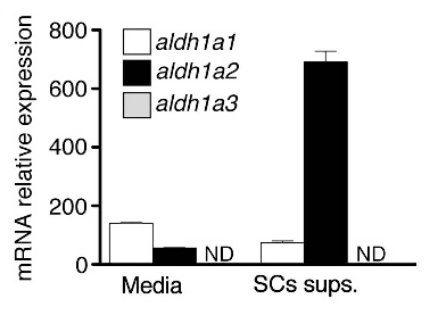

d

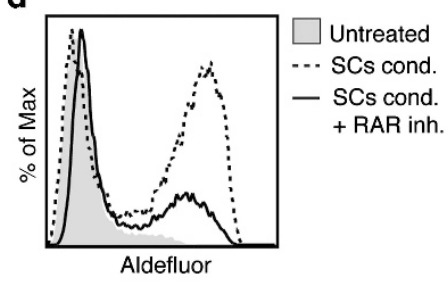

SCs cond \& DEAB

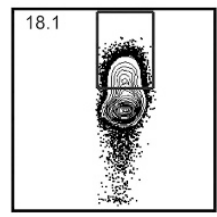

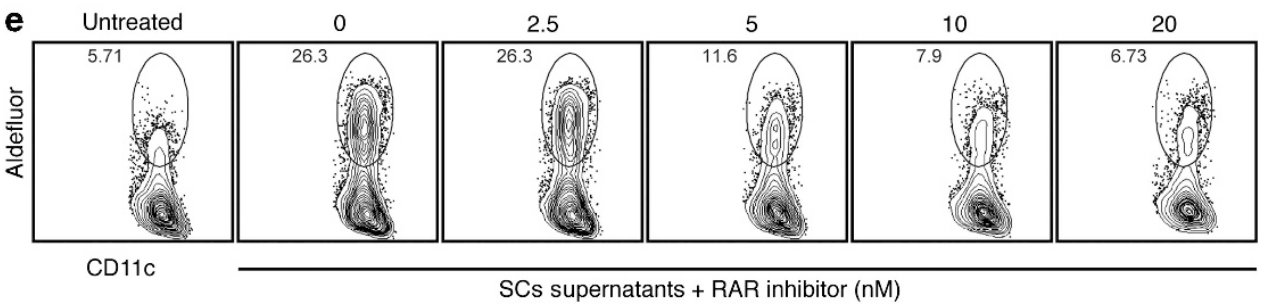

Figure 3 Dendritic cells (DCs) require retinoic acid receptor (RAR) signaling to induce RA-producing enzymes when cultured with Pdpn ${ }^{+} \mathrm{CD}^{-}$ SI LP SCs. (a) Aldefluor staining of splenic DCs (gated CD45 ${ }^{+} \mathrm{MHCI}{ }^{\text {hi }} \mathrm{CD} 11 \mathrm{c}^{+}$) after $24 \mathrm{~h}$ culture alone (untreated) or together with small intestinal (SI) stromal cells (SCs) (SCs cond.). DEAB (100 $\mu \mathrm{m}$ ) was added at the time of the staining together with aldefluor where indicated. Data show one representative experiment of $>5$ experiments with identical results. (b) Fluorescence-activated cell sorting analysis of interleukin (IL)-17A (left panel) or $\alpha 4 \beta 7$ (right panel) in paraformaldehyde/ionomycin re-stimulated OVA-specific TCR-transgenic CD4 T cells primed with splenic DCs for 4-5 days. When indicated, DEAB $(10 \mu \mathrm{m})$ was added to the DC-T-cell culture and maintained during the whole length of the experiment. Analysis shows the result from one representative experiment of at least two experiments with similar results. (c) messenger RNA (mRNA) levels of the RA-producing enzymes RALDH1 (aldh1a1), RALDH2 (aldh1a2), and RALDH3 (aldh1a3) were measured by quantitative real-time PCR in splenic DCs after $24 \mathrm{~h}$ culture in media alone or media supplemented with SC supernatants (SCs suspension (sups), obtained as in Figure 1a). Data shown correspond to RNA from three independent experiments that were quantified at the same time. (d) Aldefluor staining of splenic DCs (gated CD45 ${ }^{+} \mathrm{MHCII}^{\text {hi }} \mathrm{CD} 11 \mathrm{c}^{+}$) after $24 \mathrm{~h}$ culture alone or together with sorted SCs (SCs cond., left panel) or their supernatants (SCs sups, right panel). The global RAR global antagonist BMS204493 (RAR inh.) was added to the culture at a concentration of $20 \mathrm{~nm}$ or otherwise indicated. Data shown are from one representative experiment of at least three experiments. ND, not determined.

cultured DCs (Figure 4a). In contrast, RA added to spleen DCs cocultured with a RALDH-negative CD45- ${ }^{-} \mathrm{Pdpn}{ }^{\text {hi }}$ spleen SC line markedly increased aldefluor staining in the DCs, comparable to the level induced by the $\mathrm{RALDH}^{+} \mathrm{LP}$ SC (Figure $4 \mathrm{~b}$, left and middle panels). These data indicated that the spleen and LP SCs produced one or more factors that synergize with RA to mediate the expression of RALDH in cocultured DCs. To identify the nature of this SC-derived factor, supernatants from the aforementioned spleen SC cell line were concentrated and digested or not with proteinase $\mathrm{K}$, and used to condition spleen DCs in the presence of exogenous RA. Proteinase digestion completely abrogated the induction of 


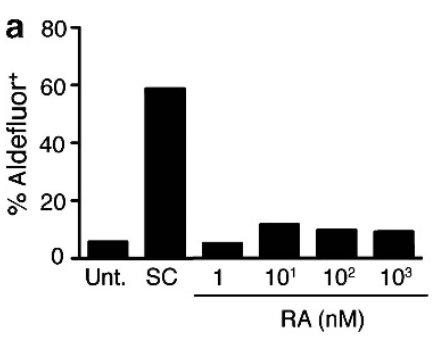

c

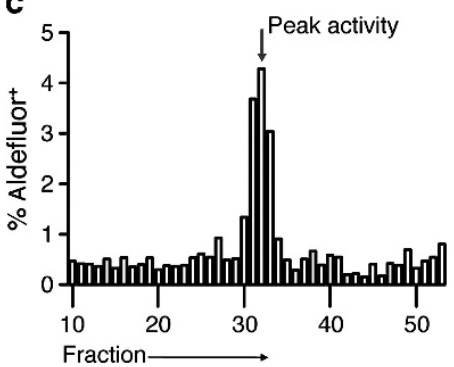

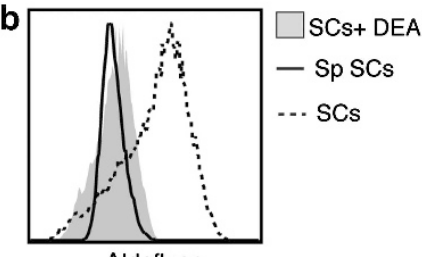

Aldefluor

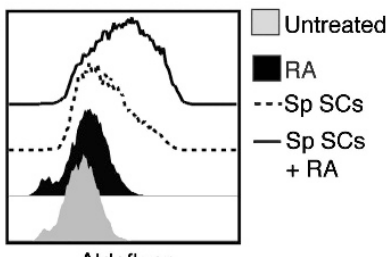

Aldefluor

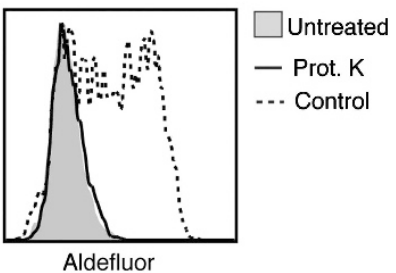

d
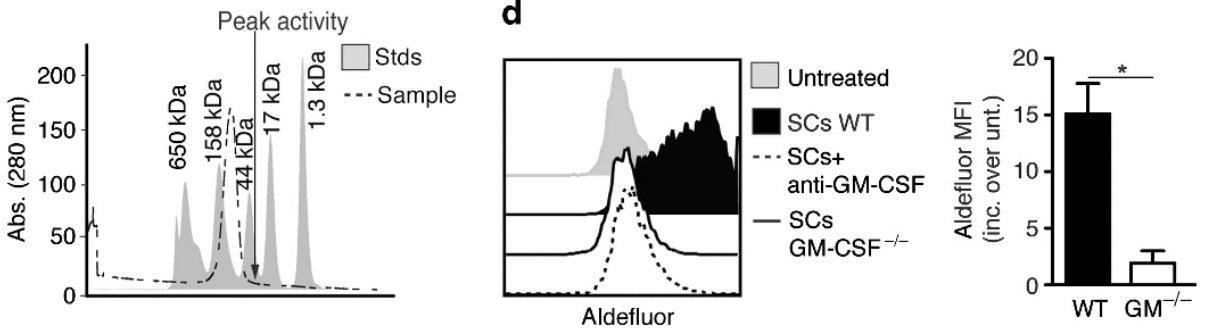

e

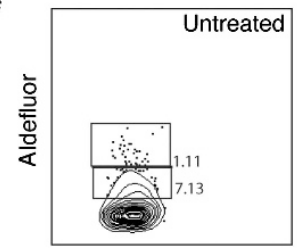

CD11c

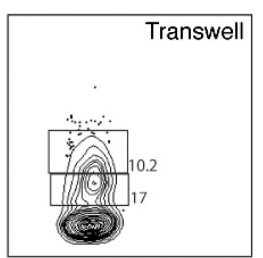

CD11c

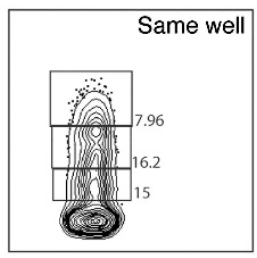

CD11c

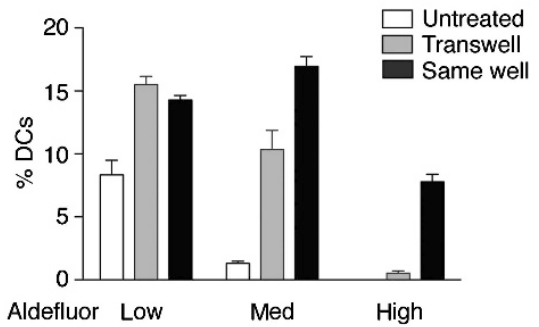

f
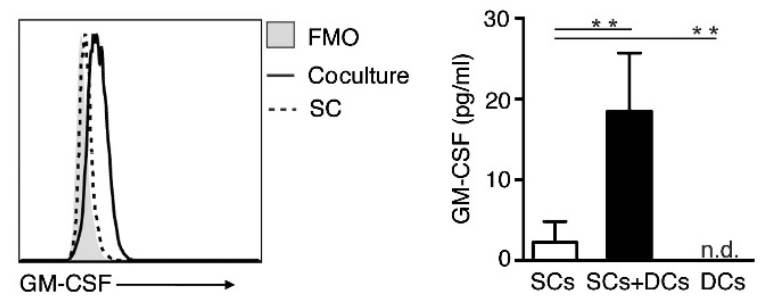

Figure 4 Granulocyte-macrophage colony-stimulating factor (GM-CSF) production by stromal cells is enhanced by dendritic cells (DCs) and required for the imprinting of DCs with high RALDH activity. (a) Aldefluor staining of splenic DCs after $24 \mathrm{~h}^{-}$treatment with SI LP CD45- Epcam ${ }^{-}$ $\mathrm{Pdpn}^{+} \mathrm{CD} 31^{-}$stromal cells (SCs) or with media supplemented with increasing concentrations of retinoic acid (RA). Data from one representative experiment of three experiments with similar results. (b) Left panel shows aldefluor staining of a cell line established from sorted primary CD45 $\mathrm{Pdpn}^{\text {hi }}$ splenic cells (Sp SCs) vs. SI LP CD45-Epcam ${ }^{-} \mathrm{Pdpn}^{+} \mathrm{CD} 31^{-}$stromal cells (SCs). Middle panel shows aldefluor staining of splenic $\mathrm{DCs}\left(\mathrm{CD} 45^{+} \mathrm{CD}_{11 \mathrm{c}}{ }^{+} \mathrm{MHCII}{ }^{\mathrm{hi}}\right.$ gate) cultured for $24 \mathrm{~h}$ with the aforementioned cell line in the presence or not of RA (100 nM). Right panel shows aldefluor staining of splenic DCs treated with RA plus concentrated suspension (sups) from the aforementioned cell line. Sups were digested with proteinase $\mathrm{K}$ when indicated. Shown is the data from one representative experiment of two. (c) Left panel shows aldefluor staining of splenic DCs after $24 \mathrm{~h}$ treatment with RA plus sequential fractions of the sups from Sp SCs (fractionation details within the Methods section). Right panel shows an estimate of the size of the proteins contained within the fraction that gave us the most activity. (d) Left panel shows aldefluor staining of splenic DCs cultured for $24 \mathrm{~h}$ alone or together with SCs from wild-type (WT) or GM-CSF knockout (KO) mice. Anti-GM-CSF blocking antibody was added where indicated. Right panel shows the fold increase on aldefluor staining (mean fluorescence intensity, MFI) on splenic DCs cocultured with SI LP CD45 ${ }^{-}$Epcam ${ }^{-} \mathrm{Pdpn}{ }^{+} \mathrm{CD} 31^{-}$SCs from WT vs. GM-CSF KO mice. Data show the results from three independent experiments ${ }^{*} P<0.05$ (unpaired $t$-test) (e) Aldefluor staining of splenic DCs after $24 \mathrm{~h}$ culture with SI LP CD45-Epcam ${ }^{-}$Pdpn ${ }^{+}$CD31 ${ }^{-}$SCs in the same well or separated by a permeable membrane (transwell). Bar graphs shows data from three replicate wells within one experiment. Data show one representative experiment out of two. (f) Shows GM-CSF by intracellular staining (left panel) or enzyme-linked immunosorbent assay (right) on SI LP CD45 ${ }^{-}$Epcam ${ }^{-}$Pdpn ${ }^{+}$CD $31^{-}$SCs cultured for $24 \mathrm{~h}$ alone or together with splenic DCs. Left panel shows one representative experiment out of three. Right panel shows data analyzed corresponding to five independent experiments. ${ }^{* *} P<0.01$ (unpaired $t$-test). ND, not determined.

RALDH in the DCs, indicating that the factor(s) secreted by the SCs were of a protein nature (Figure $4 \mathbf{b}$, right panel). To characterize the protein(s), we tested various size fractions of the supernatants for their ability to synergize with exogenous RA to induce RALDH expression in spleen DCs. Biological activity was detected in size fractions around $30 \mathrm{kDa}$ (Figure 4c). The molecular size range corresponds to the size of GM-CSF, which was shown before to also be a potent inducer of RALDH in DCs. ${ }^{15}$ To test whether the factor released by the SCs was indeed GM-CSF, conditioned spleen DCs 
cocultured with either LP SCs isolated from $\mathrm{GM}^{-\mathrm{CSF}^{-1-}}$ animals or wild-type LP SCs and anti-GM-CSF-blocking antibodies, were analyzed for RALDH induction. In the absence of GM-CSF, RALDH expression in the cocultured DCs was clearly impaired, indicating that GM-CSF produced by the SCs had a critical role in the RA-dependent transcriptional activation of aldh1a2 in DCs (Figure 4d). In order to confirm that RA signaling was required to induce RALDH2 expression in DCs, even in the presence of GM-CSF, we cultured DCs with GM-CSF in serum-free media, as serum can be a source of retinoids. ${ }^{21}$ GM-CSF alone was inefficient to support the induction of RALDH2 in cultured DCs, whereas the addition of RA greatly enhanced aldefluor staining, further supporting the existence of synergy between GM-CSF and RA in DC imprinting (Supplementary Figure S3).

To address the relative importance of the direct contact observed in vivo between the $\mathrm{CD} 103^{+}$DCs and the RA- and GM-CSF-producing LP SCs, spleen DCs and Pdpn + CD31-LP SCs were cocultured either together in the same well or separated by a permeable membrane (transwell). Although, some RALDH imprinting was observed in DCs cultured under transwell conditions, the RALDH induction was much more powerful when spleen DCs were in direct contact with the SCs in the same well (Figure 4e). Interestingly, GM-CSF production by the SCs was markedly increased in these cocultures as compared with SCs cultured alone, implying that direct contact between the DCs and SCs conversely increases the ability of these SCs to produce GM-CSF (Figure 4f).

In summary, the data show that RA and GM-CSF produced by LP SCs synergize to imprint DCs with the ability to produce RA and to functionally mature. Furthermore, the in vivo observation indicating a direct contact between $\mathrm{RALDH}^{+} \mathrm{LP}$ SCs and $\mathrm{CD}_{103}{ }^{+} \mathrm{DC}$, together with the finding that this interaction regulates GM-CSF production by the SCs, suggest that a direct cross-talk between these two cell types is required to ensure local imprinting of mucosal migratory DCs, which direct tolerogenic and inflammatory immune responses in the intestine.

\section{The generation of $\mathrm{CD} 11 \mathrm{~b}{ }^{+} \mathrm{CD}_{103}{ }^{+} \mathrm{DCs}$ and their capacity to produce RA are uniquely impaired in the absence of GM-CSF}

To address the in vivo relevance of GM-CSF for the acquisition of the RA-producing capacity by SI LP DCs, we analyzed GMCSF-deficient mice. GM-CSF is known to contribute to the survival of tissue-resident DCs, ${ }^{22}$ and in agreement, we detected a marked reduction of the SI LP CD11b ${ }^{+} \mathrm{CD}_{103}{ }^{+} \mathrm{DC}$ population (Figure 5a). Surprisingly, however, $\mathrm{CD}_{11 b^{-}} \mathrm{CD} 103^{+}$ DCs were not affected and even increased in the absence of GMCSF (Figure 5a). In addition, whereas the few remaining $\mathrm{CD} 103^{+} \mathrm{CD} 11 \mathrm{~b}^{+} \mathrm{DC}$ showed impaired capacity to produce RA in the absence of GM-CSF, this capacity was not reduced in the $\mathrm{CD}_{11 b^{-}} \mathrm{CD}_{103}{ }^{+}$population (Figure $5 \mathbf{b}$ ). These findings indicate that the LP SC-cross-talk might uniquely control the generation and/or survival and functional capacity of the SI migratory $\mathrm{CD}_{103}{ }^{+} \mathrm{CD} 11 \mathrm{~b}^{+}$DC subset.

\section{DISCUSSION}

The data presented here identify a type of LP SCs as a new player in mucosal immune regulation, able to interact with local DCs and rendering them capable to produce RA. These SCs expressed cell surface markers typical of fibroblastic reticular cells, including ICAM-1, VCAM-1, and Pdpn, ${ }^{23,24}$ known to interact with integrins such as CD11b expressed by DCs. This interaction was directly demonstrated in vivo using confocal microscopy and provides strong support for a close communication between these two cell types in situ. More importantly, these SCs expressed high levels of the aldh1a1, aldh1a2, and aldh1a3 isozymes, indicating that they were capable of producing RA. Interestingly, Pdpn ${ }^{+}$SCs from MLNs, but not peripheral lymph nodes, are also capable of expressing RALDH enzymes and promoting the induction of gut homing receptors on T cells. ${ }^{25,26}$ In vivo though, it is unlikely that these MLN SCs directly contribute to the RA-dependent imprinting of the intestinal migratory DCs, which occurs locally. ${ }^{11}$ Nevertheless, it is still possible that MLNs SCs imprint local resident DCs in the $\mathrm{LN}$ or provide RA directly to $\mathrm{T}$ cells during priming. Further investigation is needed to fully understand the role of these RA-producing MLN SCs.

Intestinal LP SCs, unlike DCs, ${ }^{14,15}$ expressed RALDH enzymes constitutively and independently of Vitamin A, suggesting that these SCs might serve as a primary source of RA for the initial education and imprinting of the RA-processing machinery in migratory DC precursor cells. In support of this, splenic DCs cultured overnight with Pdpn ${ }^{+} \mathrm{CD} 31^{-}$intestinal SCs upregulated aldh1a2 expression and gained other features associated with intestinal mucosal DCs. Although independent of vitamin A, SCs depended on the microbiota for their educational activity and failed to produce RA in germ-free mice.

Gut epithelial cells also have the capacity to produce RA, ${ }^{18,19}$ and RA-controlled gene transcription has an important part in the epithelial cell differentiation and maturation. Furthermore, in vitro studies ${ }^{27}$ showed that human intestinal epithelial cells could imprint cocultured DCs through RA together with TGF $\beta$. However, the location of $\mathrm{CD}_{103}{ }^{+}$DCs within the LP separated by the basal membrane from the epithelium makes it unlikely that imprinting by intestinal epithelial cells contributes significantly to the education of migratory DCs in vivo.

Secreted RA alone is sufficient to induce RALDH2 expression in $\mathrm{DCs} ;{ }^{16,17}$ however, the drastic increase in enzyme expression in DCs cocultured with LP SCs indicated that additional factors and/or direct contact between the two cell types further promoted the functional education of the DCs. In support of this notion, we identified GM-CSF produced by LP SCs, as an important cofactor in this education. Nevertheless, the role of GM-CSF in the functional imprinting of mucosal DCs has been controversial. ${ }^{15,28}$ Our data here show a significant loss of the $\mathrm{CD} 11 \mathrm{~b}^{+} \mathrm{CD} 103^{+} \mathrm{DC}$ subset in the absence of GM-CSF, whereas CD11b ${ }^{-} \mathrm{CD}_{103}{ }^{+}$DCs are increased. As a result, the total number of $\mathrm{CD} 11 \mathrm{c}^{+} \mathrm{CD} 103^{+} \mathrm{DCs}$ did not change in GM-CSF-deficient conditions as compared with 

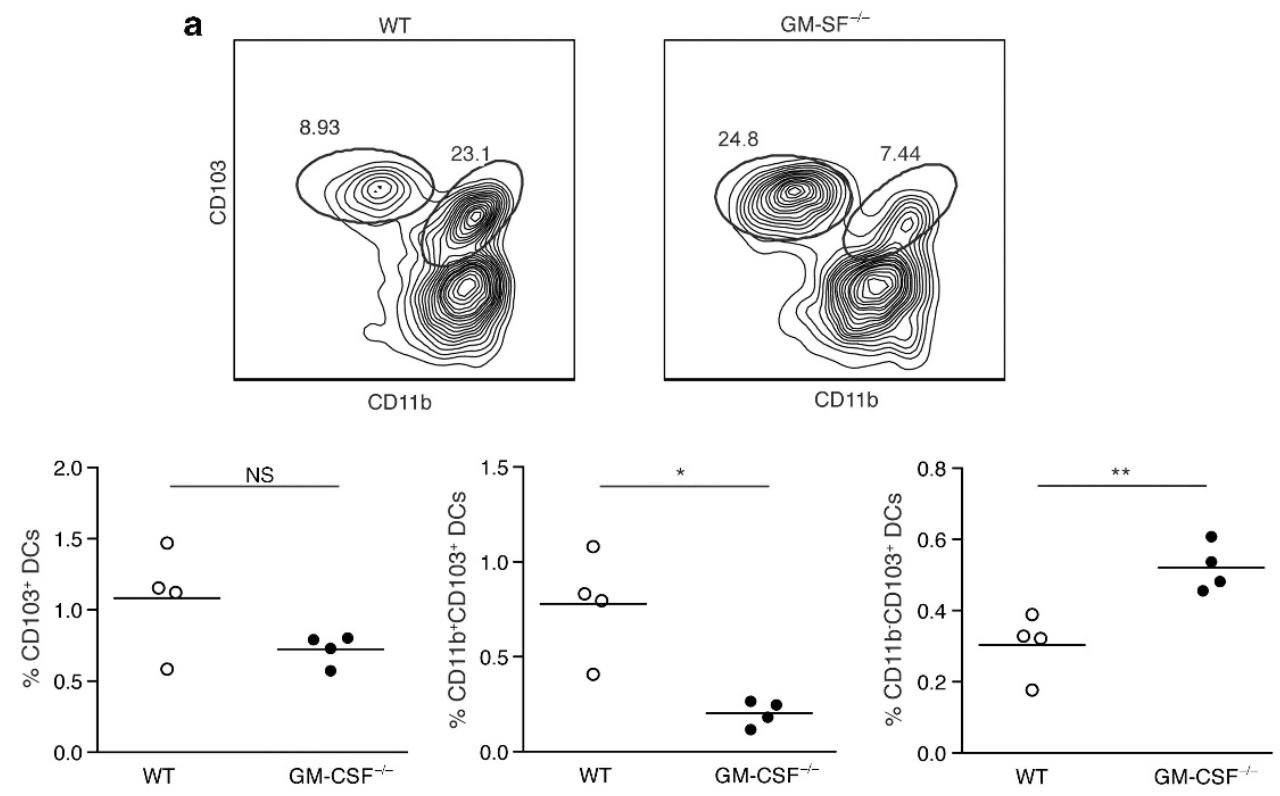

b
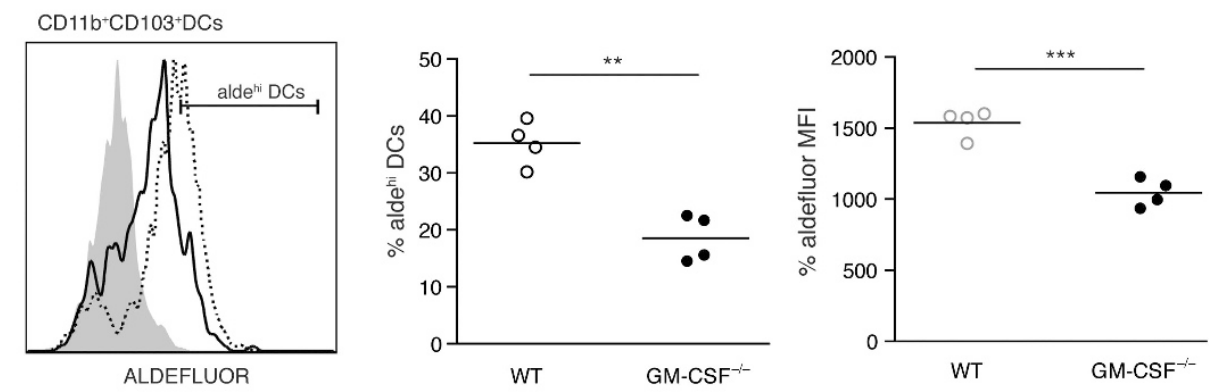

DEAB -. WT - GM-CSF ${ }^{-1-}$

CD11b-CD103+DCs
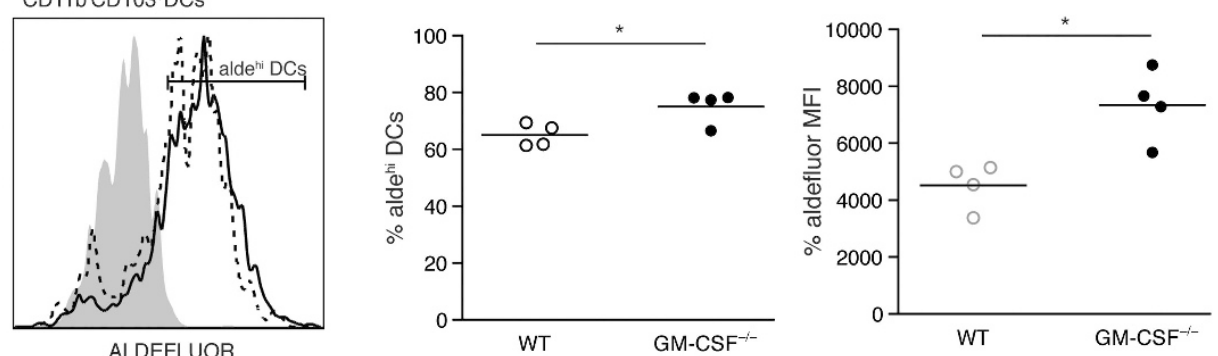

Figure $5 \mathrm{CD} 11 \mathrm{~b}^{+} \mathrm{CD}_{103}{ }^{+}$dendritic cell (DC) numbers and retinoic acid (RA)-producing capacity are diminished in granulocyte-macrophage colonystimulating factor (GM-CSF) ${ }^{-1}$ mice. (a) Upper row shows CD11b vs. CD103 staining of small intestinal (SI) lamina propria (LP) DCs (gated as CD45 ${ }^{+}$ $\mathrm{CD} 11 \mathrm{c}^{\text {hi }} \mathrm{MHCI}{ }^{\text {high }}$ ) in wild-type (WT) vs. GM-CSF knockout (KO) mice. Lower row shows the percentage of the indicated populations among the total number of SI LP cells in WT vs. GM-CSF KO mice, left panel total CD $103^{+}$DCs, middle panel CD11 $b^{+} \mathrm{CD}_{103}{ }^{+} \mathrm{DCs}$, and right panel CD11b ${ }^{-}$CD103 ${ }^{+}$ DCs. (b) Aldefluor staining of the CD11 ${ }^{+}$(upper row) and CD11b ${ }^{-}$(lower row) SI LP CD $103^{+}$DC population in WT vs. GM-CSF KO mice indicating the percentage of aldefluor high cells (middle panel) as well as the aldefluor mean fluorescence intensity (MFI) for the whole population (right panel). Data show one representative experiment of $>3$ performed (NS, not significant. $P>0.05,{ }^{\star} P<0.05,{ }^{\star \star} P<0.001,{ }^{\star \star \star} P<0.0005$ ).

wild-type mice. However, the few remaining $\mathrm{CD} 11 \mathrm{~b}^{+} \mathrm{CD} 103^{+}$ DCs in the GM-CSF ${ }^{-1-}$ animals, showed impaired capacity to produce $\mathrm{RA}$, whereas the increased subset of $\mathrm{CD} 11 \mathrm{~b}^{-} \mathrm{CD} 103^{+}$ DCs showed no defect. Therefore, when all CD11c ${ }^{+} \mathrm{CD} 103^{+}$ cells of GM-CSF knockout animals are analyzed as one pool, no noteworthy impairment in the capacity to produce RA can be detected. In contrast, when the $\mathrm{CD} 11 \mathrm{~b}^{+} \mathrm{CD} 103^{+}$subset is analyzed separately, a significant defect is evident, suggesting that GM-CSF is required for the generation and/or survival ${ }^{22}$ and imprinting of the $\mathrm{CD} 11 \mathrm{~b}^{+} \mathrm{CD}_{103}{ }^{+} \mathrm{DC}$ subset, whereas GM-CSF is not required for the development or the imprinting of the $\mathrm{CD}_{11 b^{-}} \mathrm{CD}_{103}{ }^{+}$DCs. The unique effect of GM-CSF produced by the LP SCs to control the generation and education of the SI $\mathrm{CD}_{103}{ }^{+} \mathrm{CD} 11 \mathrm{~b}^{+}$DC subset, but not the $\mathrm{CD} 103^{+}$ 
$\mathrm{CD} 1 \mathrm{~b}^{-}$DC, indicate that different SI LP DC populations might be educated through different mechanisms. This model could also explain the presence of SI LP CD103 ${ }^{+}$DCs with an RA-producing capacity observed in GF mice even when this capacity was impaired in LP SCs. It is possible that SC-derived GM-CSF has a role in the induction of the transcription factor IRF4, which is uniquely required for the development and function of the SI migratory $\mathrm{CD}_{103}{ }^{+} \mathrm{CD} 11 \mathrm{~b}{ }^{+}$DC subset. ${ }^{29}$

GM-CSF, together with RA, is also absolutely required for the education of fully differentiated DCs, indicating that GMCSF might be necessary to sensitize mature DCs, recruited to the LP from elsewhere, to RA imprinting. Interestingly, GM-CSF production by the SCs was significantly increased when fully differentiated DCs were added to the culture, suggesting that a direct two-way cross-talk initiates and promotes the unique functional education of DCs that are in close proximity of the LP SCs. This has important implications, not only for the education of local mucosal migratory DCs at steady state, but also for the re-education of newly recruited peripheral or bone marrow-derived mature DCs that migrate to the intestine in response to an inflammatory trigger. The result of SC-mediated imprinting and functional education might ameliorate or exacerbate inflammation, as RA in combination with regulatory cytokines, such as TGF- $\beta$ or inflammatory cytokines, such as IL-15 can either promote tolerance and the generation of Foxp3 Tregs or potentiate the inflammatory immune response by generating pathogenic $\mathrm{CD} 8 \alpha \beta$ and $\mathrm{CD} 4$ effector cells. ${ }^{9}$ Furthermore, the unique effect of GM-CSF, to selectively affect the generation and function of the $\mathrm{CD} 103^{+} \mathrm{CD} 11 \mathrm{~b}^{+}$DC subset, could also suggest an important role for the SI LP SCs in controlling the IRF4-dependent, IL- 6 production by the $\mathrm{CD}_{11 \mathrm{~b}^{+}}{ }^{+} \mathrm{DCs}$ important for the intestinal Th17 cell differentiation. ${ }^{29,30}$ Therefore, the SCs identified here, might be key factors in directing and controlling the immune response at the mucosal forefront. The fact that the SCs depend on the presence of the microflora for their educational role, further suggests that they are important sentinels that have the capacity to report on the condition of the intestinal lumen and accordingly regulate the tolerogenic or inflammatory nature of the immune response.

\section{METHODS}

Mice. C57BL/6 and OT-II TCR-transgenic were purchased from the Jackson Laboratories (Bar Harbor, ME). GM-CSF knockout mice were generously provided by Dr Whitsett (Cincinnati Children's Research Foundation). Mice were maintained under specific pathogen-free conditions at the La Jolla Institute for Allergy and Immunology vivarium. Animal care and experimentation were consistent with the National Institutes of Health guidelines and were approved by the Institutional Animal Care and Use Committee at the La Jolla Institute for Allergy and Immunology. Swiss Webster germ-free mice and age and gender-matched controls were purchased from Taconic Farms (Germantown, NY).

Generation of VAD mice. To generate VAD mice, pregnant females received either a chemically defined diet that lacked vitamin A (AIN$93 \mathrm{M})$ or control diet containing retinyl acetate $\left(25,000 \mathrm{IU} \mathrm{kg}^{-1}\right)$ (Dyets Inc, Bethlehem, PA). VAD diet started at 7-10 days of gestation and the pups were weaned at 3 weeks of age and maintained on the same diet at least until 11 weeks of age before analysis was performed.

\section{Epithelial cell removal and single-cell preparation from intestinal} LP. To obtain LP SCs and DCs, small intestines were removed, washed, and cut into 2-3-inche pieces. To remove the epithelial layer, these pieces were placed in Hank's balanced salt solution 5\% fetal bovine serum $0.5 \mathrm{M}$ EDTA and shaken at 250 r.p.m. for $5 \mathrm{~min}$ at $37^{\circ} \mathrm{C}$, and vortex for $1 \mathrm{~min}$ at room temperature; the tissue pieces were recovered using a stainless steel sieve, the buffer was discarded, and this process was repeated three times. The remaining intestinal tissue was minced and digested using collagenase type IV (Sigma Aldrich, St Louis, MO) for $20 \mathrm{~min}$ at $37^{\circ} \mathrm{C}$. Released cells were pelleted by centrifugation, washed with Hank's balanced salt solution $5 \%$ fetal bovine serum and passed through a $70-\mu \mathrm{m}$ in order to obtain a single cell suspension. These cells were stained for the markers Epcam, CD45, Podoplanin, and CD31 for SC sorting and CD45, MHCII, CD11c, CD11b, and CD103 for DC sorting.

Analysis of RALDH activity by aldefluor staining. RALDH activity in individual cells was analyzed using the aldefluor staining kit (StemCell Technologies, Vancouver, BC, Canada). Briefly, $1 \times 10^{6}$ cells were resuspended in the kit Assay Buffer containing activated aldefluor substrate $(150 \mathrm{~nm})$ and incubated for $30 \mathrm{~min}$ at $37^{\circ} \mathrm{C}$ in the presence or absence of the RALDH inhibitor DEAB $(100 \mu \mathrm{M})$. Afterward cells were washed, placed on ice, and stained for surface markers.

Real-Time PCR. Total RNA was extracted using TriZol reagent (Qiagen, Valencia, CA), and complementary DNA obtained with the iScript cDNA synthesis kit (Bio-Rad, Hercules, CA). Target messenger RNA was quantified using SYBR green (Roche, Applied Science, Indianapolis, IN)) and gene expression normalized relative to glyceraldehyde 3-phosphate dehydrogenase. Data were collected and analyzed on a LightCycler 380 (Roche). Primers used were GAPDH fwd 5'-ATGGCCTTCCGTGTTCCTAC-3', GAPDH rev 5'-AGATGCCTG CTTCACCAC-3', Aldh1a1 fwd 5'-TGTGGGAATACCGTGGTTG TC-3', Aldh1a1 rev 5'-GTGAAGAGCCGTGAGAGGAG-3', Aldh1a2 fwd 5'-GTGGGAGAGTGTTCCCTGTCT-3', Aldh1a2 rev 5'-TGCC TTGTCTATATCCACCTTGT-3', Aldh1a3 fwd $5^{\prime}$-GAGCAGCAA TTTCCTCCCATC-3', Aldh1a3 rev 5'-GAGCCGGTGAAGGCTAT CT-3', Rara fwd 5'-TCCGAAGAGATAGTACCCAGC-3', Rara rev 5'-AAAGCAAGGCTTGTAGATGCG-3', Rarb fwd 5' -AAGTGCTTT GAAGTGGGCAT-3', Rarb rev 5' -CTCTGTGCATTCCTGCTTTG-3' Rarg fwd 5' -AAGTACACCACGAACTCCAGT-3', Rarg rev 5'-TTCG CAAACTCCACAATCTTGA-3'.

In vitro T-cell stimulation with DCs. Splenic DCs were obtained by collagenase type IV digestion (Sigma Aldrich) $\left(1 \mathrm{mg} \mathrm{ml}^{-1}\right)$ plus DNase I $40 \mathrm{U} \mathrm{ml}^{-1}$ (Roche) and the single-cell suspension was enriched using anti-CD11c beads and MACS columns (Miltenyi Biotec, Auburn, CA); for some experiments cell were afterward sorted as $\mathrm{CD} 11 \mathrm{c}+\mathrm{MHCII}^{\mathrm{hi}}$ cells. When DCs were cocultured with SCs, $\mathrm{CD}_{4}{ }^{-}$Epcam ${ }^{-}$Podoplanin ${ }^{+} \mathrm{CD} 31^{-}$cells were sorted the day before the splenic DC preparation and cultured overnight in Iscove's modified Dulbecco's media supplemented with $10 \%$ fetal calf serum, $55 \mu \mathrm{M}$ 2-mercaptoethanol (Life Technologies-Gibco BRL, Grand Island, NY) and antibiotics (antibiotic-antimycotic, Gibco Laboratories Ltd, New York, NY) (density $25 \times 10^{3}$ cells per well in a 96-well flat-bottom plate). After overnight culture, basically all cells sorted are attached to the plate and display a fibroblast-like appearance. DCs are cocultured with SCs for $24 \mathrm{~h}$. At this time DCs were harvested from the coculture by gently washing the wells, a procedure that released the majority of DCs while the SCs remained attached to the plate. OVA-specific TCRtransgenic CD4 T cells were isolated as in Mucida et al. ${ }^{6} \mathrm{DCs}$ and T cells were plated following a 1: 5 ratio $\left(1 \times 10^{4}\right.$ DCs: $5 \times 10^{4}$ OVAspecific TCR-transgenic CD4 T cells) and OVA peptide $(1 \mu \mathrm{M})$ plus the indicated cytokines TGF $\beta\left(5 \mathrm{ng} \mathrm{ml}^{-1}\right)$ and IL-6 $\left(20 \mathrm{ng} \mathrm{ml}^{-1}\right)$ were added to the media. After 4-5 days of culture, T cells were harvested, 
washed, and expression of surface markers and cytokines was assessed by flow cytometry. Briefly, cells were incubated for $4-5 \mathrm{~h}$ with $50 \mathrm{ng} \mathrm{ml}^{-1}$ paraformaldehyde, $750 \mathrm{ng} \mathrm{ml}^{-1}$ ionomycin (both Sigma, St Louis, MO), and $10 \mu \mathrm{g} \mathrm{ml}^{-1}$ brefeldin A (Invitrogen, Carlsbad, CA) in a tissue culture incubator at $37^{\circ} \mathrm{C}$. After surface staining, cells were resuspended in fixation/permeabilization solution (BD Cytofix/ Cytoperm kit, BD, Pharmingen, San Diego, CA), and intracellular cytokine staining performed according to the protocol in this kit.

Immunofluorescence staining. For in situ aldefluor staining, intestinal explants were washed three times in Hank's balanced salt solution $5 \%$ fetal bovine serum $0.5 \mathrm{M}$ EDTA for $10 \mathrm{~min}$ at $37^{\circ} \mathrm{C}$ and shaking at 250 r.p.m. in order to remove the epithelial layer. Afterward, tissues were incubated in aldefluor assay buffer together with aldefluor reagent $(1.5 \mu \mathrm{M})$ in the presence or absence of the RALDH inhibitor DEAB $(100 \mu \mathrm{M})$. After that, tissues were washed, placed on ice, and stained for surface markers.

RA measurement using F9-RARE-lacZ reporter cell line. Sorted $\mathrm{CD} 45^{-}$Epcam ${ }^{-}$Podoplanin ${ }^{+} \mathrm{CD} 31^{-}$cells were cultured overnight in Iscove's modified Dulbecco's media supplemented with $10 \%$ fetal calf serum, $55 \mu \mathrm{M}$ 2-mercaptoethanol (Life Technologies-Gibco) and antibiotics (antibiotic-antimycotic, Gibco) (density $25 \times 10^{3}$ cells per well in a 96-well flat-bottom plate). Supernatants were stored at $-80^{\circ} \mathrm{C}$ and measurement of retinoids performed as in Petkovich ${ }^{3}$ and Wagner $^{31}$. Briefly, F9-RARE-lacZ reporter cell line cells were grown in gelatinized flasks in Dulbecco's modified Eagle's media supplemented with $15 \%$ fetal bovine serum and $0.8 \mathrm{mg} \mathrm{ml}^{-1}$ geneticin (G418) media. Once cells were near confluency they were detached from the flask using trypsin/EDTA (Gibco). Cells were washed and resuspended at 2 million cells $\mathrm{ml}^{-1}$ density and plated in a flat-bottom 96-well tissue culture plate at $2 \times 10^{5}$ cells per well $(100 \mu \mathrm{l}$ from initial dilution) plus $100 \mu \mathrm{l}$ media, different concentrations of RA or cell supernatants. After overnight culture, supernatants were removed and cells were washed extensively with ice cold phosphate-buffered saline. Following, cells were lysed and $\beta$-galactosidase activity was assayed using the $\beta$-galactosidase enzyme assay system with reporter lysis buffer from Promega (Madison, WI). Values obtained from known RA concentrations were used to draw a standard curve to quantify RA levels on the cell supernatants.

Supernatant fractionation. Supernatants were subjected to anion exchange chromatography (AEC, GE Healthcare, Niskayuna, NY; MonoQ 5/50 GL, cat \#17-5166-01) and 1-ml fractions were collected along an increasing gradient of sodium chloride $(0-1 \mathrm{M})$. Fractions were used to treat DCs $(50 \mu \mathrm{l}$ per well in a 96-well plate, total volume per well $200 \mu \mathrm{l}$ ) and the fraction that stimulated the highest RALDH2 upregulation was subjected to size exclusion chromatography, $0.5 \mathrm{ml}$ fractions were collected and used as before to stimulate DCs.

SUPPLEMENTARY MATERIAL is linked to the online version of the paper at http://www.nature.com/mi

\section{ACKNOWLEDGMENTS}

We thank Dr Noelle for providing us with the F9-RARE-lacZ cell line, Dr Whitsett for the GM-CSF knockout mice, and Dr Buckley for the Pdpn ${ }^{\text {hi }}$ splenic SC line. We thank C. Kim and K.v. Gunst for cell sorting and M. Cheroutre for her contribution. This work was supported by NIH R01 grants: R01Al050265 and DP1OD006433.

\section{AUTHOR CONTRIBUTIONS}

I.V.-S. designed and performed experiments, analyzed data, and contributed in the writing of the manuscript. A.L. and C.R. performed experiments, discussed the results and implications, and edited the manuscript. M.M., S.F., and Y.O. performed experiments and analyzed results. R.G. performed experiments. Y.W.-Z. and C.L. provided technical assistance. S.P.S., D.Z. and M.K. gave conceptual advice. H.C. designed experiments, discussed the results and their implications, supervised the experiments, and wrote the manuscript.

\section{DISCLOSURE}

The authors declared no conflict of interest.

c) 2015 Society for Mucosal Immunology

\section{REFERENCES}

1. Mark, M., Ghyselinck, N.B. \& Chambon, P. Function of retinoid nuclear receptors: lessons from genetic and pharmacological dissections of the retinoic acid signaling pathway during mouse embryogenesis. Annu. Rev. Pharmacol. Toxicol. 46, 451-480 (2006).

2. Napoli, J. Interactions of retinoid binding proteins and enzymes in retinoid metabolism. Biochim. Biophys. Acta 1440, 139-162 (1999).

3. Petkovich, M. Regulation of gene expression by vitamin A: the role of nuclear retinoic acid receptors. Annu. Rev. Nutr. 12, 443-471 (1992).

4. Iwata, M. Retinoic acid imprints gut-homing specificity on T Cells. Immunity 21, 527-538 (2004).

5. Mora, J.R. et al. Generation of gut-homing IgA-secreting B cells by intestinal dendritic cells. Science 314, 1157-1160 (2006).

6. Mucida, D. et al. Reciprocal $\mathrm{TH} 17$ and regulatory $\mathrm{T}$ cell differentiation mediated by retinoic acid. Science 317, 256-260 (2007).

7. Coombes, J. et al. A functionally specialized population of mucosal CD103 + DCs induces Foxp3 + regulatory T cells via a TGF-beta and retinoic acid-dependent mechanism. J. Exp. Med. 204, 1757-1764 (2007).

8. Benson, M.J., Pino-Lagos, K., Rosemblatt, M. \& Noelle, R.J. All-trans retinoic acid mediates enhanced $T$ reg cell growth, differentiation, and gut homing in the face of high levels of co-stimulation. J. Exp. Med. 204, 1765-1774 (2007).

9. DePaolo, R.W. et al. Co-adjuvant effects of retinoic acid and IL-15 induce inflammatory immunity to dietary antigens. Nature 471, 220-224 (2011).

10. Jaensson, E. et al. Small intestinal CD103 + dendritic cells display unique functional properties that are conserved between mice and humans. J. Exp. Med. 205, 2139-2149 (2008).

11. Johansson-Lindbom, B. et al. Functional specialization of gut CD103+ dendritic cells in the regulation of tissue-selective T cell homing. J. Exp. Med. 202, 1063-1073 (2005).

12. Agace, W. \& Persson, E. How vitamin A metabolizing dendritic cells are generated in the gut mucosa. Trends Immunol. 33, 42-48 (2012).

13. Cassani, B., Villablanca, E., De Calisto, J., Wang, S. \& Mora, R. Vitamin A and immune regulation: role of retinoic acid in gut-associated dendritic cell education, immune protection and tolerance. Mol. Aspects Med. 33, 63-76 (2012).

14. Molenaar, R. et al. Expression of retinaldehyde dehydrogenase enzymes in mucosal dendritic cells and gut-draining lymph node stromal cells is controlled by dietary vitamin A. J. Immunol. 186, 1934-1942 (2011).

15. Yokota, A. et al. GM-CSF and IL-4 synergistically trigger dendritic cells to acquire retinoic acid-producing capacity. Int. Immunol. 21, 361-377 (2009).

16. Hammerschmidt, S.I. et al. Retinoic acid induces homing of protective Tand B cells to the gut after subcutaneous immunization in mice. J. Clin. Invest. 121, 3051-3061 (2011).

17. Villablanca, E.J. \& Mora, J.R. MyD88 and retinoic acid signaling pathways interact to modulate gastrointestinal activities of dendritic cells. Gastroenterology 141, 176-185 (2011).

18. Bhat, P.V. Retinal dehydrogenase gene expression in stomach and small intestine of rats during postnatal development and in vitamin A deficiency. FEBS Lett. 426, 260-262 (1998)

19. Frota-Ruchon, A., Marcinkiewicz, M. \& Bhat, P.V. Localization of retinal dehydrogenase type 1 in the stomach and intestine. Cell Tissue Res. 302, 397-400 (2000).

20. Wagner, M., Han, B. \& Jessell, T.M. Regional differences in retinoid release from embryonic neural tissue detected by an in vitro reporter assay. Development 116, 55-66 (1992).

21. Kane, M.A., Folias, A.E. \& Napoli, J.L. HPLC/UV quantitation of retinal, retinol, and retinyl esters in serum and tissues. Anal. Biochem. 378, 71-79 (2008).

22. Greter, M. et al. GM-CSF controls nonlymphoid tissue dendritic cell homeostasis but is dispensable for the differentiation of inflammatory dendritic cells. Immunity 36, 1031-1046 (2012). 
23. Mueller, S. \& Germain, R. Stromal cell contributions to the homeostasis and functionality of the immune system. Nat. Rev. Immunol. 9, 618-629 (2009).

24. Roozendaal, R. \& Mebius, R. Stromal cell-immune cell interactions. Ann Rev. Immunol. 29, 23-43 (2011).

25. Hammerschmidt, S. et al. Stromal mesenteric lymph node cells are essential for the generation of gut-homing T cells in vivo. J. Exp. Med. 205, 2483-2490 (2008).

26. Molenaar, R. etal. Lymph node stromal cells support dendritic cell-induced gut-homing of T Cells. J. Immunol. 183, 6395-6402 (2009).

27. Iliev, I.D., Mileti, E., Matteoli, G., Chieppa, M. \& Rescigno, M. Intestinal epithelial cells promote colitis-protective regulatory T-cell differentia- tion through dendritic cell conditioning. Mucosal Immunol. 2, 340-350 (2009).

28. Wang, S. et al. MyD88-dependent TLR1/2 signals educate dendritic cells with gut-specific imprinting properties. J. Immunol. 187, 141-150 (2011).

29. Agace, W.W. IRF4 transcription-factor-dependent CD103 + CD11b + dendritic cells drive mucosal T helper 17 cell differentiation. Immunity 38 , 958-969 (2013).

30. van de Laar, L., Coffer, P.J. \& Woltman, A.M. Regulation of dendritic cell development by GM-CSF: molecular control and implications for immune homeostasis and therapy. Blood 119, 3383-3393 (2012).

31. Wagner, M.A. Use of reporter cells to study endogenous retinoid sources in embryonic tissues. Methods Enzymol. 282, 98-107 (1997). 